RESEARCH ARTICLE

\title{
Efficacy of Newer Insecticides Against Whitefly, Bemisia tabaci (Hemiptera: Aleyrodidae) on Cotton
}

Sadhana V*, Senguttuvan K, Murugan M and Sathiah N

Department of Agricultural Entomology, Tamil Nadu Agricultural University, Coimbatore 641003.

\begin{abstract}
The study aimed to evaluate the comparative efficacy of six newer insecticides as a foliar application against the whitefly, Bemisia tabaci on cotton variety C017 in two seasons' field experiments from december 2020 to july 2021 at Department of Cotton, Tamil Nadu Agricultural University, Coimbatore. Insecticides were sprayed when the whitefly population reached the economic threshold level (ETL) i.e., 4-5/3 leaf. The results revealed that flonicamid 50\% WG @ 75 g a.i. ha ${ }^{-1}$ recorded 81.99 \% reduction in mean population of whiteflies after three rounds of spray at fifteen days interval. The order of relative efficacy of test insecticides based on the mean population of whiteflies were, flonicamid 50\% WG 75 g a.i. ha ${ }^{-1}>$ dinotefuran $20 \%$ SG @ 25 g a.i. ha ${ }^{-1}>$ clothianidin 50\% WDG @ 25 g a.i. ha ${ }^{-1}>$ thiamethoxam 25 \% WG @ 50 g a.i. ha ${ }^{-1}>$ pyriproxyfen $10 \%$ EC @ 50 g a.i. ha ${ }^{-1}>$ diafenthiuron 50\% WP @ 300 g a.i. ha ${ }^{-1}>$ untreated control. Flonicamid 50\% WG @ $75 \mathrm{~g}$ a.i. ha ${ }^{-1}$ recorded increased yield of $2380 \mathrm{~kg} \mathrm{ha}^{-1}$ and $2040 \mathrm{~kg} \mathrm{ha}^{-1}$ over untreated control (1865 $\left.\mathrm{kg} \mathrm{ha}^{-1} \& 1570 \mathrm{~kg} \mathrm{ha}^{-1}\right)$ in first and second experiment, respectively.
\end{abstract}

Keywords: Newer insecticides; Bioefficacy; Whitefly; Bemisia tabaci; Cotton; Flonicamid

\section{INTRODUCTION}

The whitefly, Bemisia tabaci (Hemiptera: Aleyrodidae), occupies a significant place in the cotton crop and causes extensive damage. $B$. tabaci was not considered a severe pest of cotton in India until 2013. However, from 2014 to 2015, it became widespread and severe outbreak was reported in cotton at north western India. It caused severe damage to cotton crop in Punjab, registering a lint yield reduction from $574 \mathrm{~kg} \mathrm{ha}^{-1}$ to $197 \mathrm{~kg}$ ha $^{-1}$ from the years 2014-15 to 2015-2016 (Dhillon and Sidhu, 2016). The whitefly has various genetic groups, including Asia-I, Asia-I-India, Asia II-1, Asia II-5, Asia II-7, Asia II-8, and Asia II-11 are reported in India (Crowder et al., 2010; Ellango et al., 2015). Among these, Asia-I and Asia-II-1 were dominant in the northern cotton growing zone of India (Ellango et al., 2015). Whitefly causes indirect yield loss in the cotton crop (81.4 to $88.4 \%$ ) by transmitting cotton leaf curl disease in the northern cotton growing zone of India (Balakrishnan et al., 2009; Sain et al., 2020; Monga and Sain, 2021). Repeated applications of selective insecticides with similar modes of action may result in the development of resistance and less effective control (Saha and Mukhopadhyay, 2013). To overcome the problems of resistance, identification of new chemical molecules with better insecticidal properties, lower dosage with selective action is a continuous process for integration in Integrated Pest Management strategies. Among the new generation of insecticide molecules, flonicamid and clothianidin are new molecules with novel modes of action that are comparatively safer for the environment and economically viable for managing sucking pests in different crop ecosystems (Chandi et al., and suri, 2016). Thus, chemical control is the most essential and excellent means of control strategy for managing cotton whitefly.

\section{MATERIAL AND METHODS}

Two season experiments were conducted at the research farm, Department of Cotton, Tamil Nadu Agricultural University, Coimbatore, from december 2020 to july 2021 in Cotton C017 variety (120 days duration compact type for high-density planting) was sown during the first week of December 2020 and second week of April of first and second seasons, respectively. The experiment was laid out with seven treatments, three replications in a Randomized Block design with a plot size of $5 \times 4 \mathrm{~m}^{2}$ for each replication. The crop was raised with a spacing of 100 $x 10 \mathrm{~cm}$ following all the recommended agronomic practices of TNAU. The insecticides viz., flonicamid (Ulala 50\% WG, United Phosphorus Limited), dinotefuran (Token 20\% SG, Indofil Industries Ltd.), clothianidin (Dantotsu 50\% WDG, Sumitomo 
Chemical India Ltd), thiamethoxam (Actara 25\% WG, Syngenta India Limited), pyriproxyfen (Lano 10\% EC, Sumitomo Chemical India Ltd), diafenthiuron (Polo 50\% WP, Syngenta India Limited) were used. Pesticides were applied at 45, 60 and 75 days after sowing using a power-operated knapsack sprayer with hollow cone nozzle. Water was sprayed in the control treatment. Pre-treatment ( 0 day) and posttreatment observations were made by following the standard procedure for whiteflies. In each treatment and in each replication, five plants were selected randomly and tagged. The number of whiteflies per plant was counted on top, middle and bottom leaves on 0 and 7 days after the spray.

\section{Statistical analysis}

The corrected per cent reduction over untreated check was calculated by Henderson and Tilton (1955) formula,

Corrected per cent reduction $=\left(1-\left(\left(\mathrm{T}_{\mathrm{a}} \mathrm{X} \mathrm{C}_{\mathrm{b}}\right) /\left(\mathrm{T}_{\mathrm{b}}\right.\right.\right.$ $\left.\left.X C_{a}\right)\right) \times 100$

where,

$T_{a}$ - number of insects in the treatment after spraying

$T_{b}$ - number of insects in the treatment before spraying

$\mathrm{C}_{\mathrm{a}}$ - number of insects in the untreated check before spraying

$C_{b}$ - number of insects in untreated check after spraying

The population count was subjected to statistical analysis adopting randomized block design. The total numbers of insect population were converted to square root values. The mean values of treatments were then separated by Duncan's Multiple Range Test (DMRT) (Gomez and Gomez, 1984).

\section{RESULTS AND DISCUSSION}

The results revealed that the average whitefly population before spraying varied from 7.62 to 7.92 and was on par in statistical analysis. Whitefly population was 3.89 nos./3 leaves at 7 DAT in first spray of flonicamid 50\% WG 75 g a.i. ha-1, which was on par with dinotefuran $20 \%$ S 25 g a.i. ha ${ }^{-1}$ (3.99 nos./3 leaves) and clothianidin 50\% WDG $25 \mathrm{~g}$ a.i. ha-1 ${ }^{-1} 4.08$ nos./3 leaves) followed by 5.50 nos./3 leaves in the whitefly population in the plots treated with diafenthiuron 50\% WP 300 g a.i. ha 1 , pyriproxyfen $10 \%$ EC 50 g a.i. ha-1 (5.21 nos./3 leaves) and thiamethoxam 25\% WG 50 g a.i. ha-1 (4.45 nos./3 leaves) (Table.1). After the second spray at 7 DAT, the lowest whitefly population of 0.71 nos./3 leaves was recorded in flonicamid 50\% WG @ $75 \mathrm{~g}$ a.i. ha ${ }^{-1}$, which was on par with dinotefuran $20 \%$ S 25 g a.i. ha-1 (1.10 nos./3 leaves) and clothianidin
50\% WDG 25 g a.i. ha-1 (1.23 nos./3 leaves). The highest population of Bemisia tabaci was recorded in the plots treated with diafenthiuron 50\% WP $300 \mathrm{~g}$ a.i. ha-1 ${ }^{-1}$ (2.31 nos./3 leaves), followed by pyriproxyfen 10\% EC 50 g a.i. ha-1 (1.97 nos./3 leaves) and thiamethoxam 25\% WG 50 g a.i. ha-1 (1.47 nos./3 leaves) in decreasing order. The third round of spray recorded the highest whitefly population reduction of 0.04 nos./3 leaves in the flonicamid 50\% WG $75 \mathrm{~g}$ a.i. ha- ${ }^{-1}$ at 7 DAT, followed by dinotefuran $20 \%$ S 25 g a.i. ha-1 (0.05 nos./3 leaves) and clothianidin 50\% WDG 25 g a.i. ha-1 (0.06 nos./3 leaves). The highest population of Bemisia tabaci was recorded in the plots treated with diafenthiuron 50\% WP $300 \mathrm{~g}$ a.i. ha $^{-1}$ (0.36 nos./3 leaves), followed by pyriproxyfen 10\% EC 50 g a.i./ha-1 (0.20 nos./3 leaves) and thiamethoxam 25\% WG 50 g a.i. ha-1 $(0.10$ nos./3 leaves) in decreasing order. The highest overall mean reduction over control was recorded at plots treated with flonicamid 50\% WG 75 g a.i. ha ${ }^{-1}$ (80.79 $\%)$. It was shown to be the most effective at 7 DAT and it was statistically on par with dinotefuran $20 \%$ S 25 g a.i. ha-1 (77.52\%), followed by clothianidin 50\% WDG 25 g a.i. ha ${ }^{-1}$ (75.46\%). Thiamethoxam 25\% WG 50 g a.i. ha ${ }^{-1}$ and pyriproxyfen 10\% EC 50 g a.i. ha ${ }^{-1}$ were found to be moderately effective, with a $73.26 \%$ \& $69.54 \%$ reduction in whitefly population after 7 days of spray, respectively application. Diafenthiuron 50\% WP $300 \mathrm{~g}$ a.i. ha ${ }^{-1}$ was found to be the least effective treatment in reducing the whitefly population (66.19\%) after 7 days of spraying.

The confirmation trial of the second field experiment results is presented in Table 2. The average number of whiteflies in a population before spraying varied from 8.62-8.99 nos./3 leaves. The highest mean reduction over control was recorded at plots treated with flonicamid 50\% WG 75 g a.i. ha $^{-1}(81.99 \%)$ and it was statistically on par with dinotefuran $20 \%$ S 25 g a.i. ha ${ }^{-1}$ (79.86\%), followed by clothianidin 50\% WDG 25 g a.i. ha-1 (78.17\%). Thiamethoxam 25\% WG 50 ga.i. ha ${ }^{-1}$ and pyriproxyfen $10 \%$ EC 50 g a.i. ha ${ }^{-1}$ were found to be moderately effective, with $77.18 \%$ \& $73.84 \%$ reduction in whitefly population after 7 days of insecticide application, respectively. Diafenthiuron 50\% WP $300 \mathrm{~g}$ a.i. ha ${ }^{-1}$ was found to be the least effective treatment in reducing the whitefly population (71.02\%) after 7 days of spraying. However, if the adult whitefly population in the field crosses the ETL of five individuals per 3 leaves, insecticide such as flonicamid, dinotefuran and clothianidin can be sprayed, followed by the application of an insect growth regulator, i.e., pyriproxyfen to check the nymphal population of B. tabaci. Need-based and timely applications of pesticides with newer insecticides, viz. flonicamid 50\% WG, dinotefuran 20\% SG and clothianidin 50\% WDG are effective against $B$. tabaci under field conditions. The mean

$107|10-12| 2$ 
population reduction was reported as maximum in two experiments with flonicamid 50\% WG 75 g a.i./ ha $^{-1}$ followed by dinotefuran $20 \%$ SG, clothianidin $50 \%$ WDG, thiamethoxam 25\% WG, pyriproxyfen 10\% EC and diafenthiuron 50\% WP. Pathania et al. (2020) observed similar results, which supports our findings. Kadam (2014) also reported that dinotefuran 20\% SG and clothianidin 50\% WDG were effective in suppressing $B$. tabaci in cotton, which is consistent with our findings. The same efficacy of diafenthiuron was reported by Aslam et al. (2004), Tariq et al. (2016) and Kharel et al. (2016).

Table 1. Efficacy of novel insecticides against Bemisia tabaci on cotton -Experiment I

\begin{tabular}{|c|c|c|c|c|c|c|c|c|c|}
\hline \multirow{3}{*}{ Treatment } & \multicolumn{8}{|c|}{ Whitely population (Nos./ 3 leaves) } & \multirow{3}{*}{$\begin{array}{c}\text { Yield } \\
\left(\text { kg ha-1) }^{-1}\right)\end{array}$} \\
\hline & \multicolumn{2}{|c|}{ First spray } & \multicolumn{2}{|c|}{ Second spray } & \multicolumn{2}{|c|}{ Third spray } & \multicolumn{2}{|c|}{ Overall spray } & \\
\hline & PTC & 7 DAT & PTC & 7 DAT & PTC & 7 DAT & Mean & $\%$ Reduction & \\
\hline $\begin{array}{l}\text { Flonicamid 50\% WG } \\
\text { @ } 75 \text { g.a.i.ha-1 }\end{array}$ & $\begin{array}{c}7.92 \\
(2.90)^{\mathrm{a}}\end{array}$ & $\begin{array}{c}3.89 \\
(2.10)^{a}\end{array}$ & $\begin{array}{c}2.58 \\
(1.75)^{a}\end{array}$ & $\begin{array}{c}0.71 \\
(1.10)^{a}\end{array}$ & $\begin{array}{c}0.38 \\
(0.94)^{a}\end{array}$ & $\begin{array}{c}0.04 \\
(0.73)^{a}\end{array}$ & $\begin{array}{c}1.55 \\
(1.43)^{a}\end{array}$ & 80.79 & $\begin{array}{c}2380 \\
(48.79)^{a}\end{array}$ \\
\hline $\begin{array}{l}\text { Diafenthiuron } 50 \% W P \\
@ 300 \text { g.a.i.ha-1 }\end{array}$ & $\begin{array}{c}7.90 \\
(2.90)^{a}\end{array}$ & $\begin{array}{c}5.50 \\
(2.45)^{c}\end{array}$ & $\begin{array}{c}4.89 \\
(2.32)^{\mathrm{e}}\end{array}$ & $\begin{array}{c}2.31 \\
(1.68)^{d}\end{array}$ & $\begin{array}{c}1.49 \\
(1.41)^{f}\end{array}$ & $\begin{array}{c}0.31 \\
(0.90)^{d}\end{array}$ & $\begin{array}{c}2.71 \\
(1.79)^{d}\end{array}$ & 66.19 & $\begin{array}{c}1987 \\
(44.58)^{\mathrm{e}}\end{array}$ \\
\hline $\begin{array}{l}\text { Pyriproxyfen } 10 \% \mathrm{EC} \\
\text { @ } 50 \text { g.a.i.ha-1 }\end{array}$ & $\begin{array}{c}7.82 \\
(2.88)^{a}\end{array}$ & $\begin{array}{c}5.21 \\
(2.39)^{c}\end{array}$ & $\begin{array}{c}4.47 \\
(2.23)^{\text {de }}\end{array}$ & $\begin{array}{c}1.97 \\
(1.57)^{d}\end{array}$ & $\begin{array}{c}0.99 \\
(1.22)^{\mathrm{e}}\end{array}$ & $\begin{array}{c}0.20 \\
(0.84)^{c}\end{array}$ & $\begin{array}{c}2.46 \\
(1.72)^{d}\end{array}$ & 69.54 & $\begin{array}{c}1998 \\
(44.70)^{\mathrm{e}}\end{array}$ \\
\hline $\begin{array}{l}\text { Dinotefuran } 20 \% \text { SG } \\
\text { @ } 25 \text { g.a.i.ha-1 }\end{array}$ & $\begin{array}{c}7.81 \\
(2.88)^{\mathrm{a}}\end{array}$ & $\begin{array}{c}3.99 \\
(2.12)^{\mathrm{a}}\end{array}$ & $\begin{array}{c}3.41 \\
(1.98)^{b}\end{array}$ & $\begin{array}{c}1.10 \\
(1.26)^{b}\end{array}$ & $\begin{array}{c}0.47 \\
(0.98)^{b}\end{array}$ & $\begin{array}{c}0.05 \\
(0.73)^{\mathrm{a}}\end{array}$ & $\begin{array}{c}1.71 \\
(1.49)^{b}\end{array}$ & 77.52 & $\begin{array}{c}2272 \\
(47.67)^{\mathrm{b}}\end{array}$ \\
\hline $\begin{array}{l}\text { Clothianidin 50\%WDG } \\
\text { @ } 25 \text { g.a.i.ha-1 }\end{array}$ & $\begin{array}{c}7.62 \\
(2.85)^{a}\end{array}$ & $\begin{array}{c}4.08 \\
(2.14)^{\mathrm{ab}}\end{array}$ & $\begin{array}{c}3.54 \\
(2.01)^{\mathrm{bc}}\end{array}$ & $\begin{array}{c}1.23 \\
(1.32)^{\mathrm{bc}}\end{array}$ & $\begin{array}{c}0.53 \\
(1.01)^{c}\end{array}$ & $\begin{array}{c}0.06 \\
(0.75)^{a}\end{array}$ & $\begin{array}{c}1.79 \\
(1.51)^{b}\end{array}$ & 75.46 & $\begin{array}{c}2150 \\
(46.37)^{c}\end{array}$ \\
\hline $\begin{array}{l}\text { Thiamethoxam 25\%WG } \\
\text { @ } 50 \text { g.a.i.ha-1 }\end{array}$ & $\begin{array}{c}7.82 \\
(2.88)^{\mathrm{a}}\end{array}$ & $\begin{array}{c}4.45 \\
(2.22)^{\mathrm{b}}\end{array}$ & $\begin{array}{c}4.01 \\
(2.12)^{\mathrm{cd}}\end{array}$ & $\begin{array}{c}1.47 \\
(1.40)^{\mathrm{c}}\end{array}$ & $\begin{array}{c}0.73 \\
(1.11)^{d}\end{array}$ & $\begin{array}{c}0.10 \\
(0.77)^{\mathrm{b}}\end{array}$ & $\begin{array}{c}2.01 \\
(1.58)^{c}\end{array}$ & 73.26 & $\begin{array}{c}2077 \\
(45.58)^{d}\end{array}$ \\
\hline Control (Water spray) & $\begin{array}{c}7.82 \\
(2.88)^{a}\end{array}$ & $\begin{array}{c}8.01 \\
(2.92)^{d}\end{array}$ & $\begin{array}{l}8.55 \\
(3.01)^{f}\end{array}$ & $\begin{array}{c}8.81 \\
(3.05)^{\mathrm{e}}\end{array}$ & $\begin{array}{c}7.20 \\
(2.77)^{\mathrm{g}}\end{array}$ & $\begin{array}{c}6.00 \\
(2.55)^{\mathrm{e}}\end{array}$ & $\begin{array}{c}7.61 \\
(2.85)^{\mathrm{e}}\end{array}$ & 0.00 & $\begin{array}{c}1865 \\
(43.19)^{f}\end{array}$ \\
\hline S.Em & 0.014 & 0.090 & 0.214 & 0.334 & 0.336 & 0.312 & 0.123 & - & - \\
\hline CD & $\mathrm{N} / \mathrm{A}$ & 0.108 & 0.1157 & 0.1111 & 0.0291 & 0.0214 & 0.382 & - & - \\
\hline
\end{tabular}

DAT - Days after Treatment; PTC - Pre-Treatment Count

- Figures in the parentheses are $\sqrt{ }$ sqrt $\{x+0.5\}$ transformed values

- In a column, means followed by same letter(s) are not significantly different at $\mathrm{P}=0.05$ by DMRT.

Table 2. Efficacy of novel insecticides against Bemisia tabaci on cotton - Experiment II

\begin{tabular}{|c|c|c|c|c|c|c|c|c|c|}
\hline \multirow{3}{*}{ Treatment } & \multicolumn{8}{|c|}{ Whitely population (Nos./ 3 leaves) } & \multirow{3}{*}{$\begin{array}{c}\text { Yield } \\
\left(\mathrm{kg} \mathrm{ha}^{-1}\right)\end{array}$} \\
\hline & \multicolumn{2}{|c|}{ First spray } & \multicolumn{2}{|c|}{ Second spray } & \multicolumn{2}{|c|}{ Third spray } & \multicolumn{2}{|c|}{ Overall spray } & \\
\hline & PTC & 7 DAT & PTC & 7 DAT & PTC & 7 DAT & Mean & \% Reduction & \\
\hline $\begin{array}{l}\text { Flonicamid } 50 \% \text { WG } \\
\text { @ } 75 \text { g.a.i.ha }{ }^{-1}\end{array}$ & $\begin{array}{c}8.92 \\
(3.07)^{a}\end{array}$ & $\begin{array}{c}4.41 \\
(2.22)^{a}\end{array}$ & $\begin{array}{c}2.75 \\
(1.80)^{a}\end{array}$ & $\begin{array}{c}0.69 \\
(1.09)^{\mathrm{a}}\end{array}$ & $\begin{array}{c}0.31 \\
(0.90)^{a}\end{array}$ & $\begin{array}{c}0.69 \\
(0.73)^{a}\end{array}$ & $\begin{array}{c}1.71 \\
(1.49)^{a}\end{array}$ & 81.99 & $\begin{array}{c}2040 \\
(45.17)^{a}\end{array}$ \\
\hline $\begin{array}{l}\text { Diafenthiuron 50\%WP } \\
\text { @ } 300 \text { g.a.i.ha }{ }^{-1}\end{array}$ & $\begin{array}{c}8.99 \\
(3.08)^{a}\end{array}$ & $\begin{array}{c}5.55 \\
(2.46)^{f}\end{array}$ & $\begin{array}{l}3.69 \\
(2.05)^{f}\end{array}$ & $\begin{array}{c}1.85 \\
(1.53)^{c}\end{array}$ & $\begin{array}{c}1.57 \\
(1.44)^{b}\end{array}$ & $\begin{array}{c}1.85 \\
(1.02)^{b}\end{array}$ & $\begin{array}{c}2.65 \\
(1.78)^{d}\end{array}$ & 71.02 & $\begin{array}{c}1977 \\
(44.47)^{f}\end{array}$ \\
\hline $\begin{array}{l}\text { Pyriproxyfen } 10 \% \mathrm{EC} \\
\text { @ } 50 \text { g.a.i.ha }\end{array}$ & $\begin{array}{c}8.82 \\
(3.05)^{a}\end{array}$ & $\begin{array}{c}5.40 \\
(2.43)^{\mathrm{e}}\end{array}$ & $\begin{array}{c}3.54 \\
(2.01)^{\mathrm{e}}\end{array}$ & $\begin{array}{c}1.67 \\
(1.47)^{c}\end{array}$ & $\begin{array}{c}1.21 \\
(1.31)^{b}\end{array}$ & $\begin{array}{c}1.67 \\
(0.88)^{\mathrm{ab}}\end{array}$ & $\begin{array}{c}2.45 \\
(1.72)^{d}\end{array}$ & 73.84 & $\begin{array}{c}1991 \\
(44.63)^{\mathrm{e}}\end{array}$ \\
\hline $\begin{array}{l}\text { Dinotefuran } 20 \% S G \\
\text { @ } 25 \text { g.a.i.ha }{ }^{-1}\end{array}$ & $\begin{array}{c}8.81 \\
(3.05)^{a}\end{array}$ & $\begin{array}{c}4.81 \\
(2.30)^{b}\end{array}$ & $\begin{array}{c}2.99 \\
(1.87)^{b}\end{array}$ & $\begin{array}{c}0.90 \\
(1.18)^{b}\end{array}$ & $\begin{array}{c}0.42 \\
(0.96)^{\mathrm{a}}\end{array}$ & $\begin{array}{c}0.90 \\
(0.74)^{a}\end{array}$ & $\begin{array}{c}1.92 \\
(1.56)^{b}\end{array}$ & 79.86 & $\begin{array}{c}2027 \\
(45.03)^{b}\end{array}$ \\
\hline $\begin{array}{l}\text { Clothianidin 50\%WDG } \\
\text { @ } 25 \text { g.a.i.ha }{ }^{-1}\end{array}$ & $\begin{array}{c}8.62 \\
(3.02)^{a}\end{array}$ & $\begin{array}{c}4.99 \\
(2.34)^{c}\end{array}$ & $\begin{array}{c}3.11 \\
(1.90)^{c}\end{array}$ & $\begin{array}{c}0.99 \\
(1.22)^{b}\end{array}$ & $\begin{array}{c}0.49 \\
(1.00)^{\mathrm{a}}\end{array}$ & $\begin{array}{c}0.99 \\
(0.75)^{a}\end{array}$ & $\begin{array}{c}2.01 \\
(1.59)^{b}\end{array}$ & 78.17 & $\begin{array}{c}2015 \\
(44.89)^{c}\end{array}$ \\
\hline $\begin{array}{l}\text { Thiamethoxam 25\%WG } \\
\text { @ } 50 \text { g.a.i.ha }{ }^{-1}\end{array}$ & $\begin{array}{c}8.82 \\
(3.05)^{a}\end{array}$ & $\begin{array}{c}5.35 \\
(2.42)^{d}\end{array}$ & $\begin{array}{c}3.27 \\
(1.94)^{d}\end{array}$ & $\begin{array}{c}1.09 \\
(1.26)^{\mathrm{b}}\end{array}$ & $\begin{array}{c}0.55 \\
(1.02)^{a}\end{array}$ & $\begin{array}{c}1.09 \\
(0.76)^{\mathrm{a}}\end{array}$ & $\begin{array}{c}2.17 \\
(1.64)^{c}\end{array}$ & 77.18 & $\begin{array}{c}2003 \\
(44.76)^{d}\end{array}$ \\
\hline Control (Water spray) & $\begin{array}{c}8.82 \\
(3.05)^{a}\end{array}$ & $\begin{array}{c}8.88 \\
(3.06)^{g}\end{array}$ & $\begin{array}{c}8.99 \\
(3.08)^{g}\end{array}$ & $\begin{array}{c}9.10 \\
(3.10)^{\mathrm{e}}\end{array}$ & $\begin{array}{c}8.65 \\
(3.02)^{c}\end{array}$ & $\begin{array}{c}9.10 \\
(3.00)^{c}\end{array}$ & $\begin{array}{c}8.82 \\
(3.05)^{\mathrm{e}}\end{array}$ & 0.00 & $\begin{array}{c}1570 \\
(39.63)^{g}\end{array}$ \\
\hline S.Em & 0.013 & 0.004 & 0.004 & 0.028 & 0.044 & 0.048 & 0.154 & - & - \\
\hline$C D$ & $\mathrm{~N} / \mathrm{A}$ & 0.013 & 0.012 & 0.086 & 0.136 & 0.151 & 0.480 & - & - \\
\hline
\end{tabular}

- DAT - Days after Treatment; PTC - Pre-Treatment Count

- Figures in the parentheses are $\sqrt{ }$ sqrt $\{x+0.5\}$ transformed values

- In a column, means followed by same letter(s) are not significantly different at $\mathrm{P}=0.05$ by DMRT. 


\section{CONCLUSION}

The present study concluded that flonicamid $50 \%$ WG 75 g a.i. ha-1 (81.99\%) was shown to be the most effective with maximum per cent reduction of whitefly and highest yield in both experiments and it was statistically on par with dinotefuran $20 \%$ S $25 \mathrm{~g}$ a.i. ha-1. Need-based application of flonicamid $50 \%$ WG 75 g a.i. ha-1 followed by dinotefuran $20 \%$ S 25 g a.i. ha-1 may be incorporated as the chemical component in the integrated pest management strategies against whitefly.

\section{REFERENCES}

Aslam, M., Razaq, M., Shah, S.A. and F. Ahmad. 2004. Comparative efficacy of different insecticides against sucking pests of cotton. J. Res. Sci., 15(1): 53-58.

Balakrishnan, N., Kumar, B.V. and P. Sivasubramanian. 2009. Bioefficacy of bifenthrin 10 EC against sucking insects, bollworms and natural enemies in cotton. Madras Agric. J., 96(1/6): 225-229.

Biswas, K.K., Bhattacharyya, U.K., Palchoudhury, S., Balram, N., Kumar, A., Arora, R., Sain, S.K., Kumar, P., Khetarpal, R.K., Sanyal, A. and P.K. Mandal. 2020. Dominance of recombinant cotton leaf curl Multan-Rajasthan virus associated with cotton leaf curl disease outbreak in northwest India. PLoS one., 15(4): 1-19.

Chandi, R.S. and K.S. Suri. 2016. Field efficacy of newer insecticides against tomato fruit borer, Helicoverpa armigera (Hübner). Pestic. Res. J., 28(1): 15-19.

Crowder, D.W., Horowitz, A.R., De Barro, P.J., Liu, S.S., Showalter, A.M., Kontsedalov, S., Khasdan, V., Shargal, A., Liu, J. and Y. Carriere. 2010. Mating behaviour, life history and adaptation to insecticides determine species exclusion between whiteflies. J Anim Ecol., 79(3): 563-570.

Dhillon, B.S. and R.S. Sidhu. 2016. Successful management of whitefly incidence in cotton through joint efforts of farm experts and farmers. Prog. Fmg-Mont. J. Punjab Agric. Univ., 52: 5-7.

Ellango, R., Singh, S.T., Rana, V.S., Gayatri Priya, N., Raina, H., Chaubey, R., Naveen, N.C., Mahmood, R., Ramamurthy, V.V., Asokan, R. and R. Rajagopal. 2015. Distribution of Bemisia tabacigenetic groups in India. Environ. Entomol., 44(4): 1258-1264.
Gomez, K.A. and A.A. Gomez. 1984. Stat. Proc. Agric. Research. A Wiley International Science Publication, John Wiley and Sons, New York. 680p.

Henderson, C.F. and E.W. Tilton. 1955. Tests with acaricides against the brown wheat mite. J. Econ. Entomol., 48(2): 157-161.

Kadam, D. 2014. Seasonal incidence of sucking pests of Bt cotton in different intercropping systems and their natural enemies. J Pest Sci., 1(1): 29-34.

Kharel, S., Singh, P.S. and S.K. Singh. 2016. Efficacy of newer insecticides against sucking insect pests of green gram [ Vigna radiata (L.) Wilczek]. Int. j. agric. environ. biotechno., 9(6): 1081-1087.

Monga, D. and S.K. Sain. 2021. Incidence and severity of cotton leaf curl virus disease on different BG II hybrids and its effect on the yield and quality of cotton crop. J. Environ. Biol., 42(1): 90-98.

Pathania, M., Verma, A., Singh, M., Arora, P.K. and N. Kaur. 2020. Influence of abiotic factors on the infestation dynamics of whitefly, Bemisia tabaci (Gennadius 1889) in cotton and its management strategies in North-Western India. Int. J. Trop. Insect Sci., 40: 969-981.

Razaq, M., Suhail, A., Aslam, M., Arif, M.J., Saleem, M.A. and M.H.A. Khan. 2005. Evaluation of neonicotinoids and conventional insecticides against cotton jassid, Amrasca devastans (Dist.) and cotton whitefly, Bemisia tabaci (Genn.) on cotton. Pak. Entomol., 27(1): 75-78.

Saha, D. and A. Mukhopadhyay. 2013. Insecticide resistance mechanisms in three sucking insect pests of tea with reference to North-East India: an appraisal. Int. J. Trop. Insect Sci., 33(1): 46-70.

Sain, S.K., Monga, D., Mohan, M., Sharma, A. and J. Beniwal. 2020. Reduction in Seed Cotton Yield Corresponding with Symptom Severity Grades of Cotton Leaf Curl Disease (CLCUD) in Upland Cotton (Gossypium hirsutum L.). Int. J. Curr. Microbiol. App. Sci., 9(11): 3063-3076.

Tariq, K., Ali, A., Naz, G., Anwar, Z., Khan, A.A., Jan, I., Khursheed, I., Shah, J.A., Ali, K., Nadeem, M. and A. Gulzar. 2016. Field Evaluation of Yellow Sticky Traps for the Control of Citrus Whitefly Dialeurodes citri (Ashmead) (Homoptera: Aleyrodidae) in Citrus Orchard of Pakistan. J. Zool., 11(2): 110-116. 